\title{
Procedure validation and laboratory performance monitoring for the measurement of moisture, ash and volatile matter mass fractions in solid biofuels
}

\author{
Maria Trancoso $\cdot$ Ana Rita Sousa $\cdot$ Sandra Calisto
}

Received: 2 July 2014/ Accepted: 9 January 2015

(C) Springer-Verlag Berlin Heidelberg 2015

\begin{abstract}
Participation in interlaboratory comparisons is a requirement of the accreditation bodies for granting laboratory accreditation by EN ISO/IEC 17025:2005 as external quality control. Proficiency testing (PT) is used to demonstrate the individual performance of a laboratory for a specific test or measurement. Monitoring PT performance over time shows the continuing performance and allows the identification of potential problems related to random and systematic errors. In this study, it is shown that the procedures applied to measure mass fractions of moisture, ash and volatile matter in solid fuels fulfill the conditions stated in the European standards EN 14774-3, EN 14775 and EN 15148:2009 regarding target uncertainty, repeatability and reproducibility. Repeatability was assessed from sample duplicate analysis and combined standard uncertainty. Limits of detection and quantification were also estimated although no target values are stated. The obtained values fulfill the requirements for specifications and classes of solid biofuels. Laboratory performance over the time from 2011 to 2013 regarding such measurements was evaluated with 18 materials provided by WEPAL within the BIMEP program. Apart from monitoring the individual $z$-scores, their sequence was checked with summarizing parameters including the 'rescaled sum $z$-scores', RSZ, the 'sum
\end{abstract}

Presented at the Eurachem Workshop, May 2014, Lisbon, Portugal.

Electronic supplementary material The online version of this article (doi:10.1007/s00769-015-1105-8) contains supplementary material, which is available to authorized users.

M. Trancoso $(\bowtie) \cdot A$. R. Sousa $\cdot$ S. Calisto

Laboratório Nacional de Energia e Geologia, LNEG,

Unidade de Bionergia, Estrada do Paço do Lumiar, Ed. E,

1649-038 Lisbon, Portugal

e-mail: maria.trancoso@lneg.pt squared $z$-scores', SSZ, and $J$-scores. For none of the analytes, the parameters indicated a trend over time and therefore it was not necessary to trigger any investigation or correcting procedure.

Keywords Solid biofuel · Proficiency testing · Cumulative scores - Target uncertainty

\section{Introduction}

Proficiency testing (PT) is used to demonstrate the individual performance of a laboratory for a specific test or measurement procedure and to monitor the continuing performance by means of an independent assessment. Participation in interlaboratory comparisons is a requirement of the accreditation bodies for granting laboratory accreditation by EN ISO/IEC 17025 [1] as external quality control. Guidelines and standards have been developed over the last years with emphasis on harmonization protocols [2-5] and accreditation of the PT providers [5, 6]. For an accredited laboratory, it is not enough to demonstrate its performance level for its various tasks once, but the performance must be monitored over time in order to identify potential problems related to bias or trend.

In this work, measurement procedures are described which were used to determine the mass fractions of moisture, ash and volatile matter in different types of biomass. The procedures, based on standardized methods [7-9], were validated and their compliance with the performance requirements stipulated by the European standards was assessed and verified. The following performance parameters were studied: limit of detection, limit of quantification, measurement precision under 